\title{
Three-dimensional fracture mapping of multi-fragmentary patella fractures (AO/OTA 34C3)
}

\author{
Yu Zhan ${ }^{1 \#}$, Yingqi Zhang ${ }^{2 \#} \wedge$, Xuetao Xie ${ }^{1 \wedge}$, Congfeng Luo ${ }^{1 \wedge}$ \\ ${ }^{1}$ Department of Orthopaedic Surgery, Shanghai Jiao Tong University Affiliated Sixth People's Hospital, Shanghai, China; ${ }^{2}$ Department of \\ Orthopaedic Surgery, Tongji Hospital, School of Medicine, Tongji University, Shanghai, China \\ Contributions: (I) Conception and design: X Xie, Y Zhan, Y Zhang; (II) Administrative support: C Luo; (III) Provision of study materials or patients: \\ Y Zhan, X Xie; (IV) Collection and assembly of data: Y Zhan, Y Zhang; (V) Data analysis and interpretation: Y Zhan; (VI) Manuscript writing: All \\ authors; (VII) Final approval of manuscript: All authors. \\ \#These authors contributed equally to this work. \\ Correspondence to: Xuetao Xie; Congfeng Luo. Department of Orthopaedic Surgery, Shanghai Jiao Tong University Affiliated Sixth People's Hospital, \\ Shanghai, China. Email: xuetaoxie@163.com; congfengl@outlook.com.
}

Background: Multi-fragmentary patella fractures (MFPFs) are common patella fracture type. Low-profile plate fixation seems to be promising advancement in the treatment of such difficult fractures. There is no systematic morphologic study specifically for MFPFs to provide objective reference for the improvement of future implants and biomechanical models. This study aimed to delineate and quantify the location and spatial frequency of fracture lines, comminution zones, and coronal plane fragments in MFPFs using threedimensional (3D) CT mapping technique.

Methods: A total of $187 \mathrm{MFPFs}$ were retrospectively reviewed and analyzed. Fractures were digitally reconstructed from CT data, and fracture lines, comminution zones, and coronal fragments were graphically overlaid onto a 3D patella template. Fracture characteristics were summarized qualitatively based on the fracture maps and quantitatively on the counts and volume of each fragment. Furthermore, according to the classic fracture patterns concerning MFPFs, subgroup analysis was conducted.

Results: On average, we observed 7 fragments in each fracture, 3 of which were $<1 \mathrm{~cm}^{3}$. Most fractures $(81.2 \%)$ had coronal fragments on the anterior and/or posterior patella surfaces. We identified three classic patella fracture patterns: transverse with comminution, stellate, and "displaced comminuted" in 104, 54, and 29 knees, respectively. 3D maps demonstrated distinct distribution fracture patterns of fracture lines, comminution zones, and coronal fragments.

Conclusions: Supero-medial corner of the patella was seldomly involved, and might be used as the cornerstone for fixation. Coronal fragments were common on both anterior and posterior patella surfaces, justifying the application of anterior plate osteosynthesis characterized by multi-planar fixation. Comminution areas mainly concentrated in the lower half of the patella, potentially suitable for an implant in combination with sutures or circumferential cerclage wiring. The described 3D features of MFPFs could provide reference for the design of future implants and biomechanical models.

Keywords: Multi-fragmentary patella fractures; AO/OTA 34C3; 3D fracture morphology; fracture maps; patella fracture patterns

Submitted Apr 14, 2021. Accepted for publication Jul 14, 2021.

doi: $10.21037 /$ atm-21-1814

View this article at: https://dx.doi.org/10.21037/atm-21-1814

\footnotetext{
$\wedge$ ORCID: Yu Zhan, 0000-0002-7551-5039; Yingqi Zhang, 0000-0002-4616-6888; Xuetao Xie, 0000-0003-2159-0030; Congfeng Luo, 0000-0001-5876-5266.
} 


\section{Introduction}

Patella fractures account for approximately $1 \%$ of all skeletal fractures (1). Multi-fragmentary patella fractures (MFPFs) are common patella fracture type, but restoration of patellofemoral joint congruity and maintenance of stable fixation allowing for early postoperative rehabilitation remain challenging $(2,3)$. Tension band wiring has been widely used in the treatment of patella fractures, however, it is associated with poor functional outcome and surgical complications, including a high rate of implant removal (3-5). Recently, a number of studies demonstrated that low-profile plating could fix patella fractures rigorously and improve postoperative knee function, particularly in comminuted patella fracture patterns (6-8). It also should be noted that plates may be bulky and irritate soft tissues, thus increasing the risk of wound healing problems. As the fixation methods may be influenced by the patella fracture patterns and comminution areas, a systematic morphologic study specifically for MFPFs is in need to provide reference for optimal surgical strategy.

Conventional radiography in two dimensions is insufficient to demonstrate the complex fracture patterns of MFPFs. The CT based fracture mapping technique, particularly in $3 \mathrm{D}$ context, has proved useful to define the spatial features of complex fractures $(9,10)$. The purpose of this study was to delineate the location and frequency of fracture lines, comminution zones, and coronal fragments of MFPFs using the 3D mapping technique. We hypothesized that $3 \mathrm{D}$ fracture mapping would demonstrate salient features of MFPFs and that there were distinct, recurrent characteristics among different classic patterns. We present the following article in accordance with the STROBE reporting checklist (available at https://dx.doi.org/10.21037/ atm-21-1814).

\section{Methods}

\section{Subjects}

We conducted a retrospective study at a large level-I trauma center. We searched in the orthopaedic database for patients diagnosed with a patella fracture between January 2017 and April 2018. The latest AO/OTA classification was used to identify all $34 \mathrm{C} 3$ patella fractures, which refer to "complete articular, frontal/coronal, multi-fragmentary fractures" (11). We excluded patients who were without CT imaging data, skeletally immature, or had a history of knee surgery. The study was conducted in accordance with the Declaration of
Helsinki (as revised in 2013). The study was approved by Institutional Ethics Committee of Shanghai Sixth People's Hospital (No.: 2016-89-1) and individual consent for this retrospective analysis was waived.

\section{Fracture mapping}

The $3 \mathrm{D}$ fracture mapping process was conducted as described previously $(9,10)$ (Figure 1). Briefly, DICOMformatted CT data of all MFPFs were imported into Mimics software (Version 17.0, Materialise, Leuven, Belgium) for $3 \mathrm{D}$ reconstruction and segmentation of each fragment. The segmented fragments of each case were then exported to 3-matic software (Version 10.2, Materialise, Leuven, Belgium) for 3D mapping. These digitally reconstructed fractures were rotated, normalized to fit a $3 \mathrm{D}$ template of the patella. The osseous contours of the whole patella, area of the articular surface, and distal pole were anatomical landmarks referenced in the fitting process. The surface of the template was marked and divided into small independent surfaces; the borders coincided with the fracture lines of patella fragments. The strokes around the surfaces were used to delineate the fracture lines of fragments $\geq 1 \mathrm{~cm}^{3}$, while the filling color on the surfaces was used to describe the comminution area composed of fragments $<1 \mathrm{~cm}^{3}$ (Figure 1E). All fracture lines and comminution zones were overlaid onto the template $3 \mathrm{D}$ model to produce a spatial fracture map. Heat maps based on the spatial frequency of fracture lines were generated with custom E3D software (12) (Central South University, Changsha, China).

Besides the "all-in-one" fracture map, separate maps were created according to the classic patellar fracture patterns $(13,14)$. The type C3 patella fractures were classified into transverse pattern with comminution, stellate/comminuted pattern, or "displaced comminuted" pattern.

\section{Coronal plane fractures}

MFPFs can occur in multiple planes, and those containing coronal plane fractures will raise additional fixation concern (15). Therefore, special attention was given to interpret the distribution and frequency of coronal fragments $\left(\geq 1 \mathrm{~cm}^{3}\right)$.

\section{Quantitation of comminution extent}

Increased comminution of a fracture is related to increased surgical complexity and a worse outcome (16). To quantify 

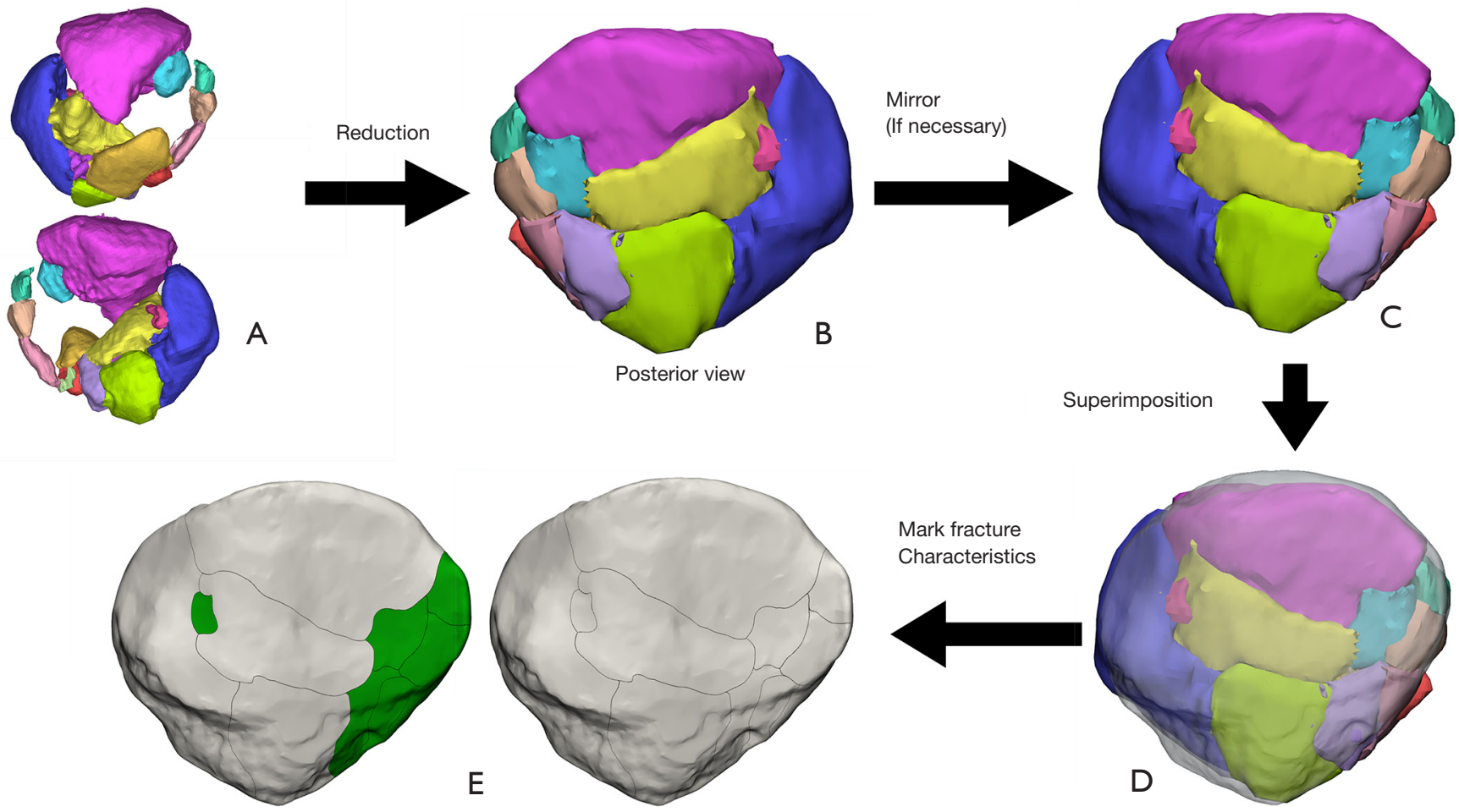

Figure 1 Schematic of method used for 3D computed tomography (CT) mapping of multi-fragmentary patella fractures. With the CT images of each fracture, patella fragments were segmented and reconstructed as independent 3D models (A), and then digitally "reduced" (B). Each reduced fractured patella was then fit to an intact 3D patella template. The osseous contours of the whole patella, the articular surface, and the distal pole were anatomical landmarks referenced in the fitting process. The patient's fracture was mirror reversed if necessary and then fit onto the model (C,D). The contour of the large fragment $\left(\geq 1 \mathrm{~cm}^{3}\right)$ is digitally delineated with thin lines (black), and the area containing small fragments $\left(<1 \mathrm{~cm}^{3}\right)$ is identified by a marked (green) zone $(\mathrm{E})$.

the comminution extent, we counted the fragments in each fracture, and calculated the average volume of larger fragments $\left(\geq 1 \mathrm{~cm}^{3}\right)$ for each fracture.

\section{Statistical analysis}

Both descriptive and quantitative analyses of fracture maps were employed. Comparisons of categorical-level data were evaluated with Chi-square tests. Fisher's exact test was used when appropriate. Continuous data of the three patterns of fractures were evaluated with one-way analysis of variance (ANOVA) and Scheffe's post-hoc tests. SPSS version 23.0 (IBM Corp., Armonk, NY, USA) was used. Significance was set at $\mathrm{P}<0.05$.

\section{Results}

Patient demographic and clinical characteristics were summarized in Table 1. A total of 187 fractures in 186 patients were included in this study. Overall, there were more MFPFs in men for most age-decades (Figure 2), and an unequal distribution of fracture patterns was found among males and females (Table 1).

All three classic fracture patterns were observed in this group of patients: (I) transverse, with comminution on one side; (II) stellate/comminuted, non-displaced; and (III) "displaced comminuted" (Table 1; Figure 3). The transverse, stellate, and "displaced comminuted" patterns accounted for $55.6 \%, 28.9 \%$, and $15.5 \%$ of all $34 \mathrm{C} 3$ fractures, respectively. The "displaced comminuted" pattern was more common in male patients than in female $(\mathrm{P}<0.01)$ (Table 1).

\section{Fracture maps}

\section{Overall distribution}

The fracture lines of the 187 MFPFs occurred frequently 
Table 1 Demographic and clinical characteristics of study sample

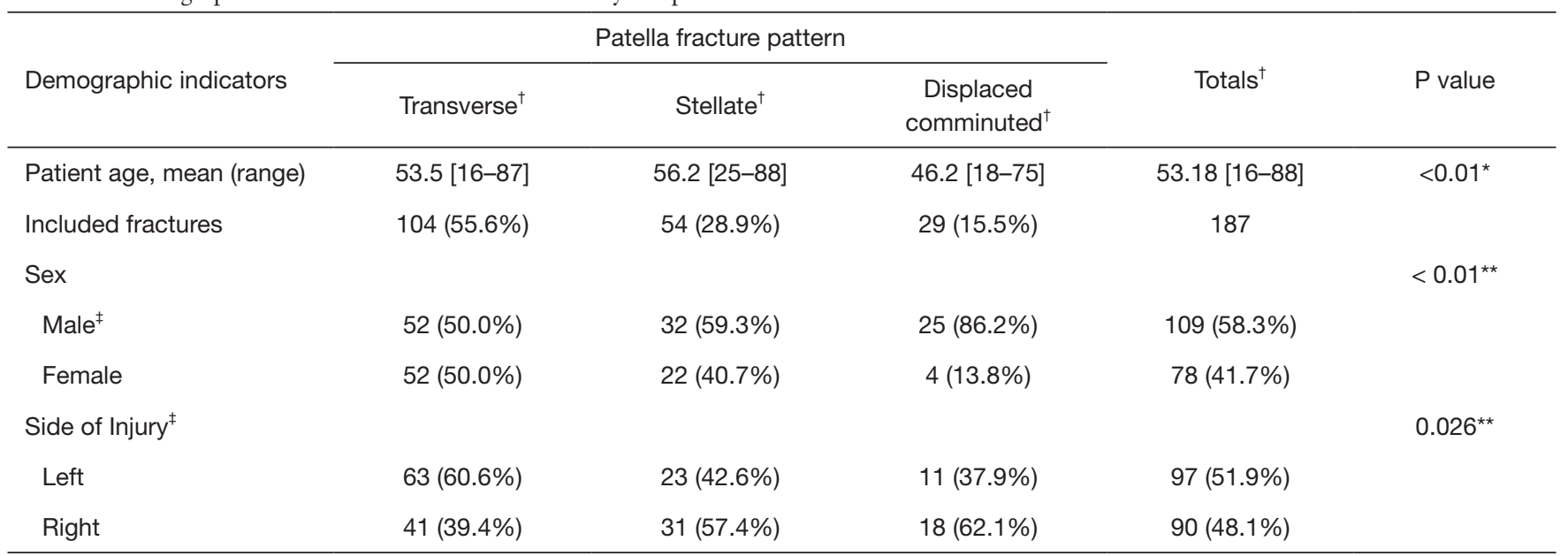

${ }^{\dagger}$, data are presented as number (percentage), except for the patient age row as the mean (range). The percentages shown are based on the total number of fractures for each fracture pattern $(n=104,=54$, or $=29$, respectively) or for the "Totals" category, on the total number of subjects $(\mathrm{N}=187) .{ }^{\ddagger}$, one male subject had bilateral multi-fragmentary patella fractures, so both fractures contributed data. *, significant differences evaluated by one-way analysis of variance (ANOVA), Scheffe's post hoc test revealed significant difference between the comminuted displaced pattern and the other two patterns. ${ }^{* *}$, chi-square test for sex and side of injury between the three patterns.

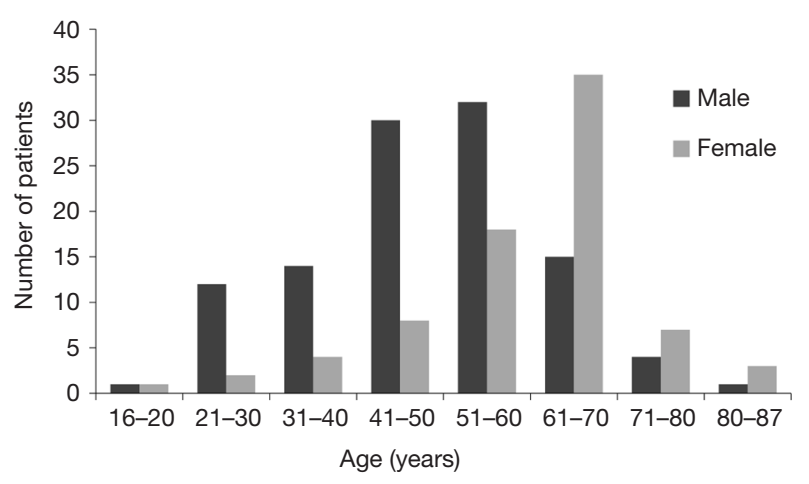

Figure 2 Frequency distribution of fractures grouped by patients' age-decade and sex.

in the distal half of the anterior patella surface (Figure 4). Posteriorly, the lines concentrated mainly in the lateralinferior quadrant and wrapped around the medial-inferior quadrant along the border of the articular surface inferiorly. In both the medial and lateral rims of the patella, the fracture lines were distributed mainly in the lower twothirds. The lower half of the lateral rim was more likely to be fractured than the medial rim. Notably, fracture lines in the superior-medial corner were least common. The comminution zones containing fragments $<1 \mathrm{~cm}^{3}$ mainly concentrated in the lower half of the patella (Figure 5).

\section{Transverse pattern}

There were 104 fractures displaying the transverse pattern (Table 1). The vast majority $(95 / 104,91.3 \%)$ had fracture lines and comminutions in the distal patella, while the proximal third of the patella was rarely involved.

\section{Stellate pattern}

There were 54 fractures in this group. The fracture lines on the anterior surface were radially distributed and tended to concentrate just below the center part of the patella. Comminution areas were distributed diffusely on both anterior and posterior surfaces of the patella, with the lateral-inferior corner having the highest frequency.

\section{"Displaced comminuted" pattern}

There were 29 fractures of this pattern, and fracture lines were highly scattered on both anterior and posterior surfaces of the patella, and fracture lines concentrated on the lateral-inferior corner. The distribution of comminution zones was similar to that of the stellate pattern.

\section{Coronal fractures}

We observed a high incidence of coronal fractures, with $81.2 \%$ of patients having coronal fragments. The incidence in each pattern was shown in Table 2. Coronal fragments were present in $100 \%$ of the "displaced comminuted" 


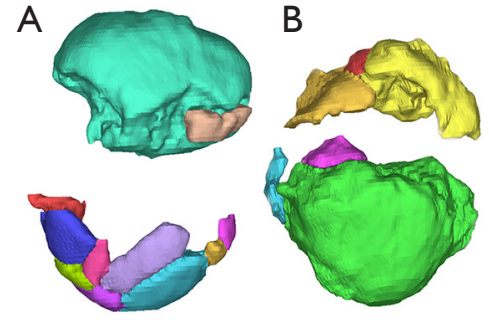

Transverse (with comminution)
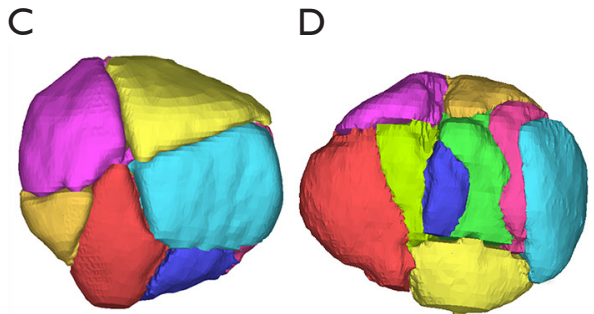

Stellate
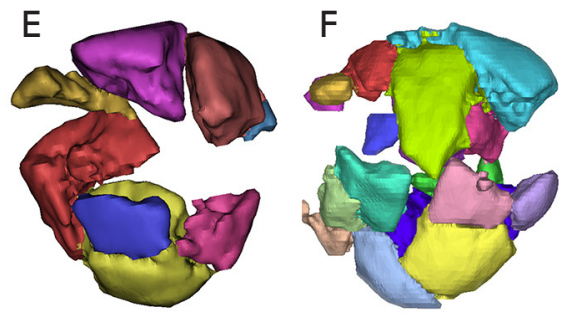

Displaced comminuted

Figure 3 Examples of the three possible classical fracture patterns of the multi-fragmentary patella fractures. Examples were reconstructed in three dimensions before reduction. The transverse pattern mostly had comminutions at the distal part of the patella (A), rarely at the proximal part (B). The comminutions of stellate fracture lines can be radially organized around the central patella (C) or have an irregular pattern with minor displacement (D). The "displaced comminuted" fracture pattern is characterized by severe comminution and displacement, but without a relatively complete side like the transverse pattern $(\mathrm{E}, \mathrm{F})$.
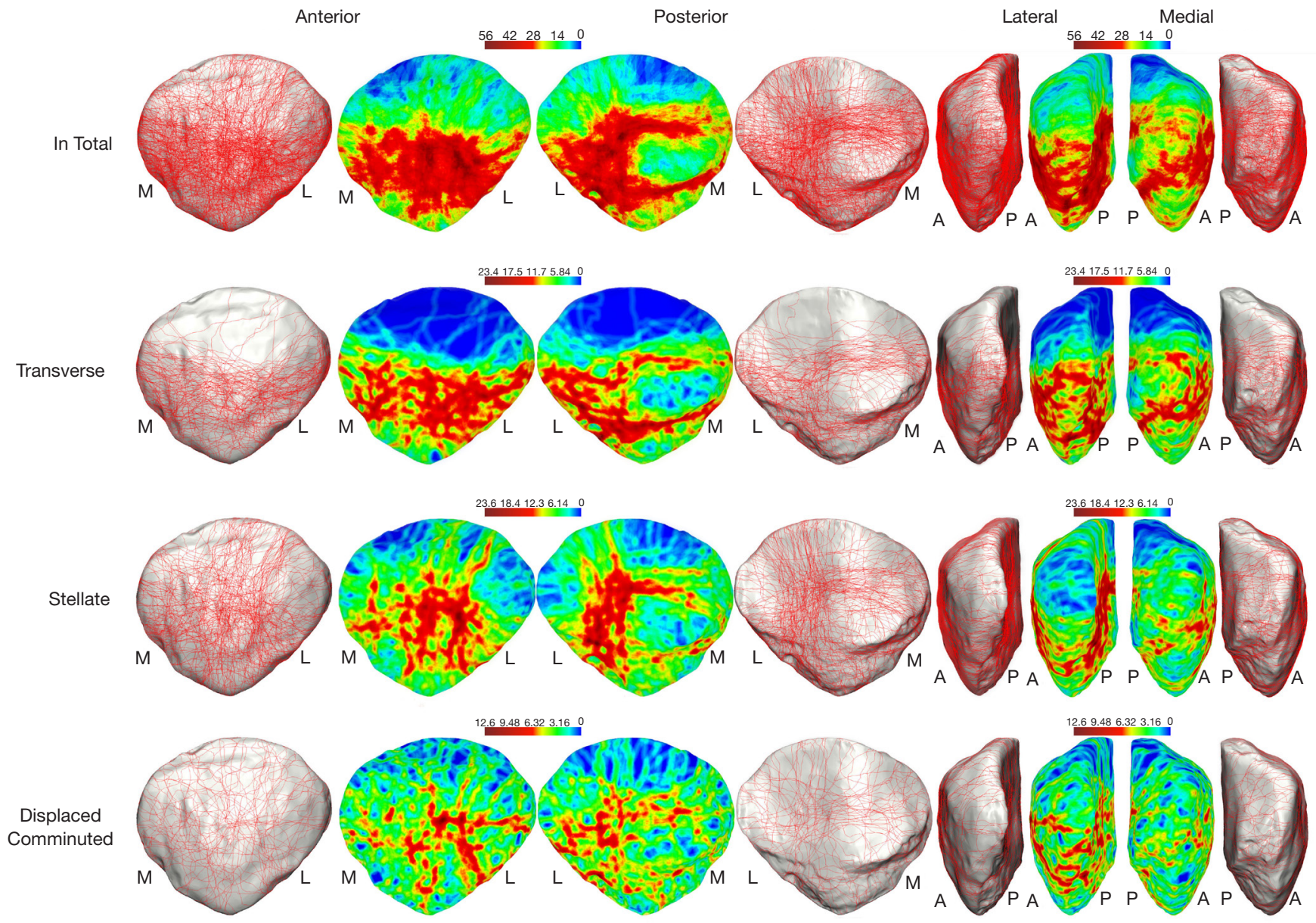

Figure 4 Spatial distribution of fracture lines of all multi-fragmentary patella fractures in this study. All fracture lines (top row) were overlaid onto a standard 3D patella model, with spatial density indicated in 3D heat maps for each pattern (top to bottom) shown in frontal and A-P views (left to right). Colored scale represents the frequency, with red indicating the highest frequency and dark blue the lowest frequency. A, $\mathrm{P}$, $\mathrm{L}$, and $\mathrm{M}$ stand for anterior, posterior, lateral, and medial side, respectively. 

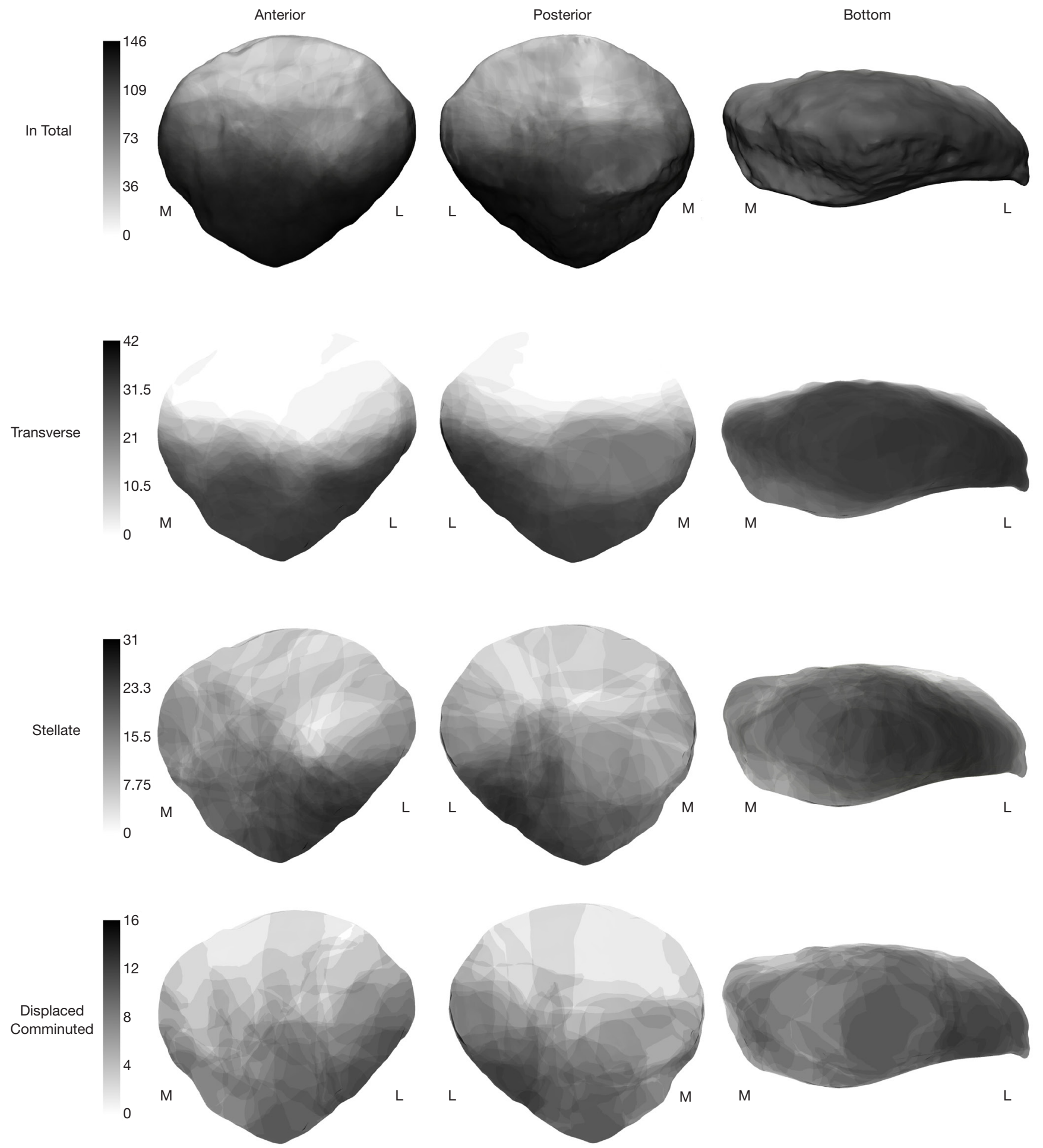

Figure 5 Spatial concentration of the comminuted fragments. Comminution zones containing fragments $<1 \mathrm{~cm}^{3}$ mainly concentrated in the lower half of patella. Overall 3D maps of small comminution zones (1st row) and maps of each pattern (top to bottom). Maps in first row are shown on 3D models for referencing anatomical landmarks. Images in second through fourth rows are presented without 3D model to eliminate obfuscation of zones caused by light reflection in 3D rendering. The gradient grayscale represents frequency, with the darkest gray representing the most frequent. A, P, L, and M key indicators same as in Figure 4. 
Table 2 Coronal-plane fracture frequency

\begin{tabular}{|c|c|c|c|c|c|}
\hline \multirow{2}{*}{$\begin{array}{l}\text { Coronal fragments } \\
\text { indicators }\end{array}$} & \multicolumn{3}{|c|}{ Patella fracture pattern } & \multirow{2}{*}{ Totals } & \multirow{2}{*}{$P$ value } \\
\hline & Transverse & Stellate & Displaced comminuted & & \\
\hline Anterior surface ${ }^{\ddagger}$ & $68(65.4 \%)$ & $30(55.6 \%)$ & 27 (93.1\%) & 120/151 (79.5\%) & $<0.01^{*}$ \\
\hline Both $^{\ddagger}$ & $27(26.0 \%)$ & $13(24.1 \%)$ & $21(72.4 \%)$ & $61 / 151(40.4 \%)$ & $<0.01^{\star}$ \\
\hline
\end{tabular}

${ }^{\dagger}$, data are presented as count/total numbers (percentage); ${ }^{\ddagger}$, the percentages shown are based on the total number of fractures for each fracture category; ${ }^{*}$, significant differences detected by Chi-square test.

pattern, and in $72.4 \%$ of this pattern, both anterior and posterior areas were involved. Figure 6 illustrated the spatial distribution of these fragments.

Overall, anterior coronal fragments concentrated mainly in the distal half of the patella, which was also observed in the transverse and stellate patterns. In the "displaced comminuted" pattern, anterior coronal fragments were diffusely distributed, while posterior fragments concentrated in the articular surface, particularly in the central area.

\section{Comminution analysis}

Overall, MFPFs had 7.3 fragments on average (Table 3). The "displaced comminuted" pattern was the most comminuted, with an average of 11.3 fragments, and was significantly more than the other patterns $(\mathrm{P}<0.01) .89 .3 \%$ of cases had fragments $<1 \mathrm{~cm}^{3}$, the "displaced comminuted" pattern had an average of 5.4 small fragments $\left(<1 \mathrm{~cm}^{3}\right)$ and 5.6 large fragments $\left(\geq 1 \mathrm{~cm}^{3}\right)$, and post-hoc tests showed that all three patterns are significantly different when compared pairwise $(\mathrm{P}<0.01)$.

The bony volume of the patella was comparable among all patterns $(\mathrm{P}=0.277)$. The average volume of larger fragments $\left(\geq 1 \mathrm{~cm}^{3}\right)$ was greatest in the transverse pattern, and this volume was significantly larger compared to that of the other two patterns $(\mathrm{P}<0.01)$.

\section{Discussion}

The $3 \mathrm{D}$ fracture mapping technique was adopted in a large series of MFPFs in order to delineate and quantify the features of AO/OTA 34C 3 fractures. On the basis of a previous study investigating 83 type $\mathrm{C}$ patella fractures (17), we focused on the most challenging patella fractures-187 type C3. This study combined qualitative maps and quantitative evaluation of coronal fragments and comminution to offer comprehensive morphological demonstration of MFPFs. The findings in this study will not only improve understanding of C3 type patella fractures during the formulation of surgical strategy, but also can potentially be used as reference for biomechanical studies and development of new fixation tools.

High prevalence of coronal fragments shown in the 3D fracture maps facilitates us to consider the current fixation strategy. In this series, $81.2 \%$ had coronal fragments and $58.9 \%$ occurred on the posterior articular surface. Conventional fixation methods (e.g., tension band wires, screws) probably could not stabilize these fragments reliably (Figure 7), as pointed out by Lazaro et al. (15). From a morphological point of view, the anterior plate osteosynthesis offering anteroposterior screw fixation may have advantages in the treatment of these fragments. The location and frequency of the coronal fragments described in this study could provide reference for the configuration of such plate-screw constructs.

Distribution of large fragments $\left(>1 \mathrm{~cm}^{3}\right)$ and comminution zone $\left(<1 \mathrm{~cm}^{3}\right)$ may also provide useful information for plate design and fixation strategy. The supero-medial corner of the patella was least involved, so it can be potentially used as the cornerstone for fracture fixation. The comminution zone containing fragments $<1 \mathrm{~cm}^{3}$ mainly concentrated in the lower half of the patella, which connects with the patellar tendon. It was reported that anterior plating established a "cage" construct allowing screw purchase in an orthogonal fashion to rigorously capture the comminuted inferior pole and the relatively large fragments in the supero-medial corner (18). A recent 


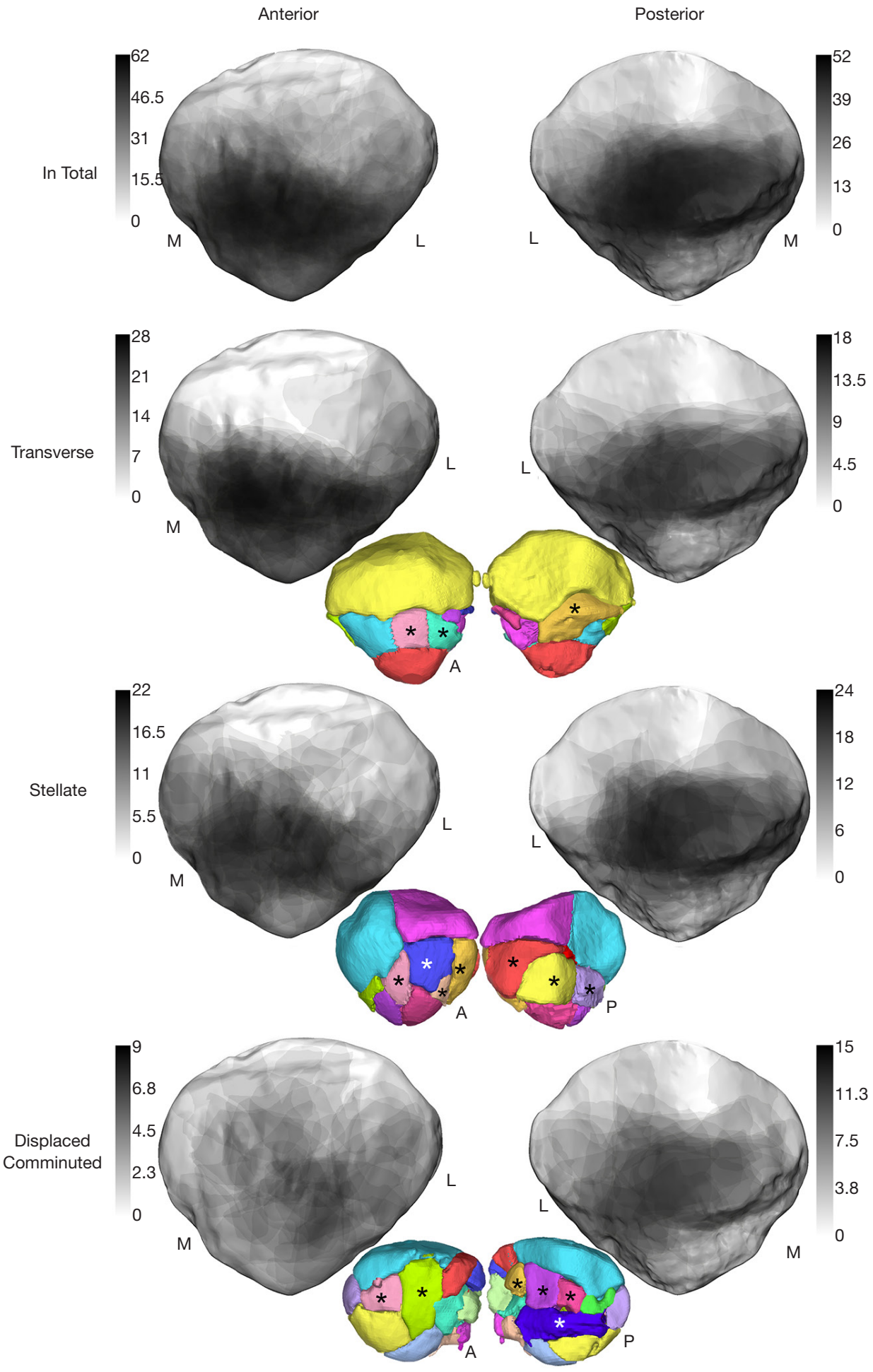

Figure 6 Spatial concentration of coronal fractures. 3D maps of coronal fragments in anterior and posterior views. Six small colored 3D models show the anterior and posterior views of reconstructed fragments from three representative patients with coronal fractures. A, P, L, and $M$ key indicators same as in Figures 4,5, and the same grayscale frequency key. Asterisks indicate coronal fragments. 
Table 3 Quantitative comminution characteristics

\begin{tabular}{|c|c|c|c|c|c|}
\hline \multirow[b]{2}{*}{ Comminution indicators } & \multicolumn{3}{|c|}{ Patella fracture pattern } & \multirow[b]{2}{*}{ Overall means $^{\dagger}$} & \multirow[b]{2}{*}{$P$ value } \\
\hline & Transverse $^{\dagger}$ & Stellate ${ }^{\dagger}$ & $\begin{array}{c}\text { Displaced } \\
\text { comminuted }^{\dagger}\end{array}$ & & \\
\hline No. of fractures with small fragments $\left(<1 \mathrm{~cm}^{3}\right)^{\ddagger}$ & 95/104 (91.3\%) & $45 / 54(83.3 \%)$ & $27 / 29(93.1 \%)$ & $167 / 187(89.3 \%)$ & $0.234^{\# \#}$ \\
\hline Count of fragments $<1 \mathrm{~cm}^{3 \#}$ & $3.2 \pm 2.0$ & $1.9 \pm 1.6$ & $5.4 \pm 2.6$ & $3.2 \pm 2.3$ & $<0.01^{\star}$ \\
\hline Patellar bony volume $\left(\mathrm{cm}^{3}\right)$ & $20.7 \pm 4.6$ & $20.6 \pm 4.9$ & $22.1 \pm 3.8$ & $20.9 \pm 4.6$ & 0.277 \\
\hline Average volume of larger fragments $\geq 1 \mathrm{~cm}^{3}\left(\mathrm{~cm}^{3}\right)$ & $5.9 \pm 2.5^{* *}$ & $4.7 \pm 1.5$ & $4.0 \pm 1.4$ & $5.3 \pm 2.2$ & $<0.01^{*}$ \\
\hline
\end{tabular}

${ }^{\dagger}$, data are given as mean \pm standard deviation, unless otherwise noted. ${ }^{\ddagger}$, data are presented as number/total fracture number of each pattern (percentage), the percentages shown are based on the total number of fractures for each fracture category $(n=104,=54$, or $=29$, respectively), or the total number of subjects $(\mathrm{N}=187)$. Statistics of the number or volume of small comminuted fragments does not include fractures without fragments $<1 \mathrm{~cm}^{3}$. ${ }^{*}$, significant differences detected by one-way ANOVA test or Chi-square test between the three patterns. **, Scheffe's post hoc test found significant differences between this pattern and the other two patterns. " , significant difference detected between any selected two patterns by Scheffe's post hoc test. ${ }^{\# \#}$, Chi-square test result.

biomechanical study found that anterior mesh plating with biplanar screw fixation was superior to antero-lateral plating and tension band wiring in fixation of comminuted patella fractures characterized by 2 superior fragments and 4 inferior fragments in each cadaveric model (18). Sutures and circumferential cerclage wiring through holes in the anterior plates may be adopted to enhance the fixation to firmly reconstruct the extensor mechanism. While there were studies reporting the use of basket plates and vertical wiring to fix the comminuted inferior pole fractures $(19,20)$, their success relied on the prerequisite that few articular fragments were involved in patella fractures. Thus, they may not be suitable for treatment of $\mathrm{C} 3$ type patella fractures.

Distinct morphology of the three fracture patterns demonstrated in this study may have different biomechanical implications. Previous biomechanical studies have examined the strength of internal fixators mainly based on the model of type $\mathrm{C} 1$ transverse patellar fractures $(21,22)$. The results could not be extrapolated directly into MFPFs. Recently, a 6-part patella fracture model was artificially created to mimic the presence of the coronal fragments and comminution in the lower part of patella fractures (18). As for the stellate pattern fractures, fracture lines on the anterior surface were radially distributed. Wagner et al. created such fractures in their biomechanical study and found that anterior plating provided significantly better primary stability in comparison with modified tension band wiring with cannulated screws (23). Among the 3 patterns, the "displaced comminuted" fracture pattern was the most comminuted involving every corner of the patella. Circumferential cerclage wiring may be helpful before the application of an anterior locking plate able to capture maximal fragments in multi-planar fixation fashion.

This study has some limitations. Firstly, unlike mapping of simple fractures, main fracture lines were not explored in this study due to the nature of MFPFs. Secondly, since the morphologic evaluation of patella fractures was based on CT data, the fracture maps may not represent those fractures without CT images. Finally, functional outcomes and treatment complications were not included in the analysis of these challenging fractures, thus correlation between fracture patterns and clinical prognosis could not be established.

\section{Conclusions}

In conclusion, this study elucidated the morphology of MFPFs (AO/OTA 34C3) based on a large series of patella fractures in 3D maps. Overall, fracture lines, comminution zones, and coronal fragments mainly concentrated in the lower half of the patella. Supero-medial corner was least involved, and thus may be used as reference for fracture reduction and fixation. Coronal fragments were frequent on both anterior and posterior patella surfaces, justifying 

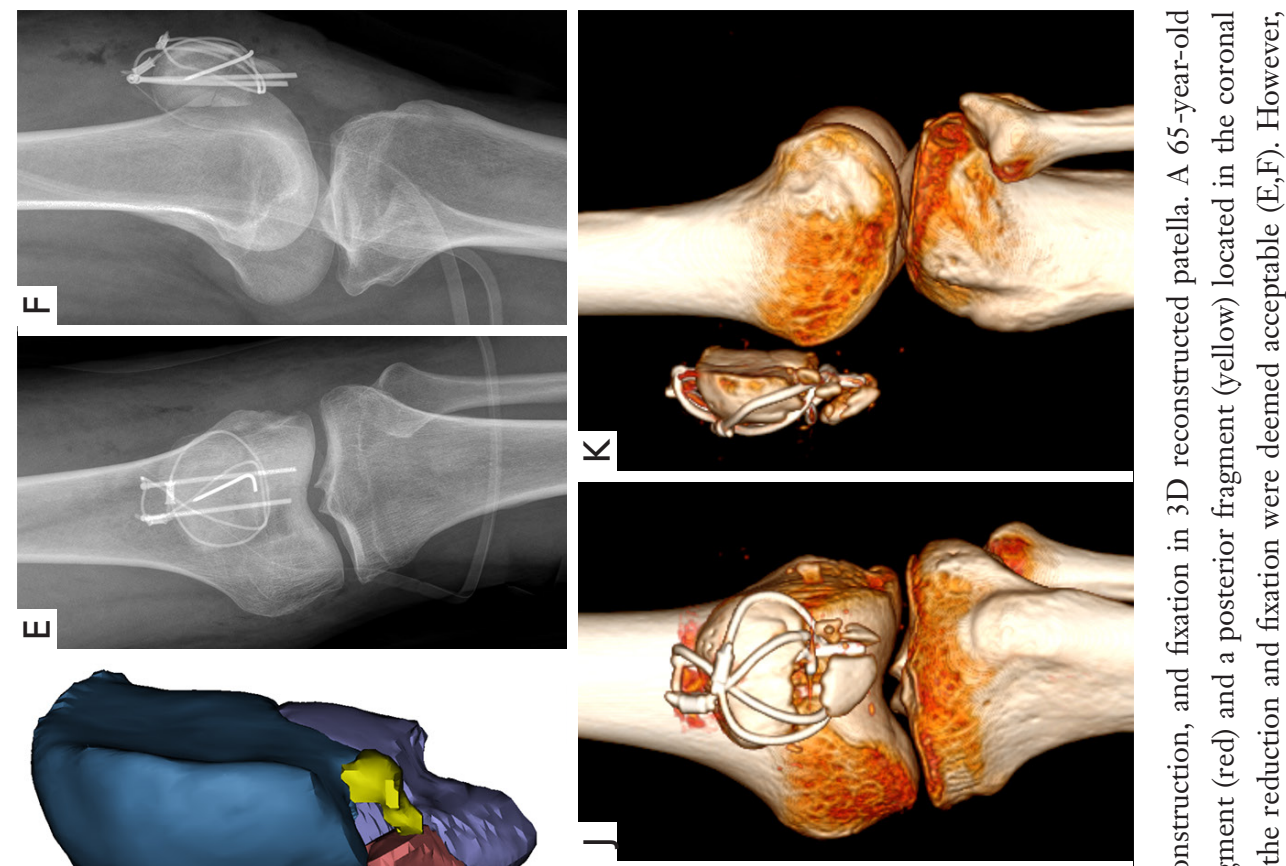

कि

:

绨造
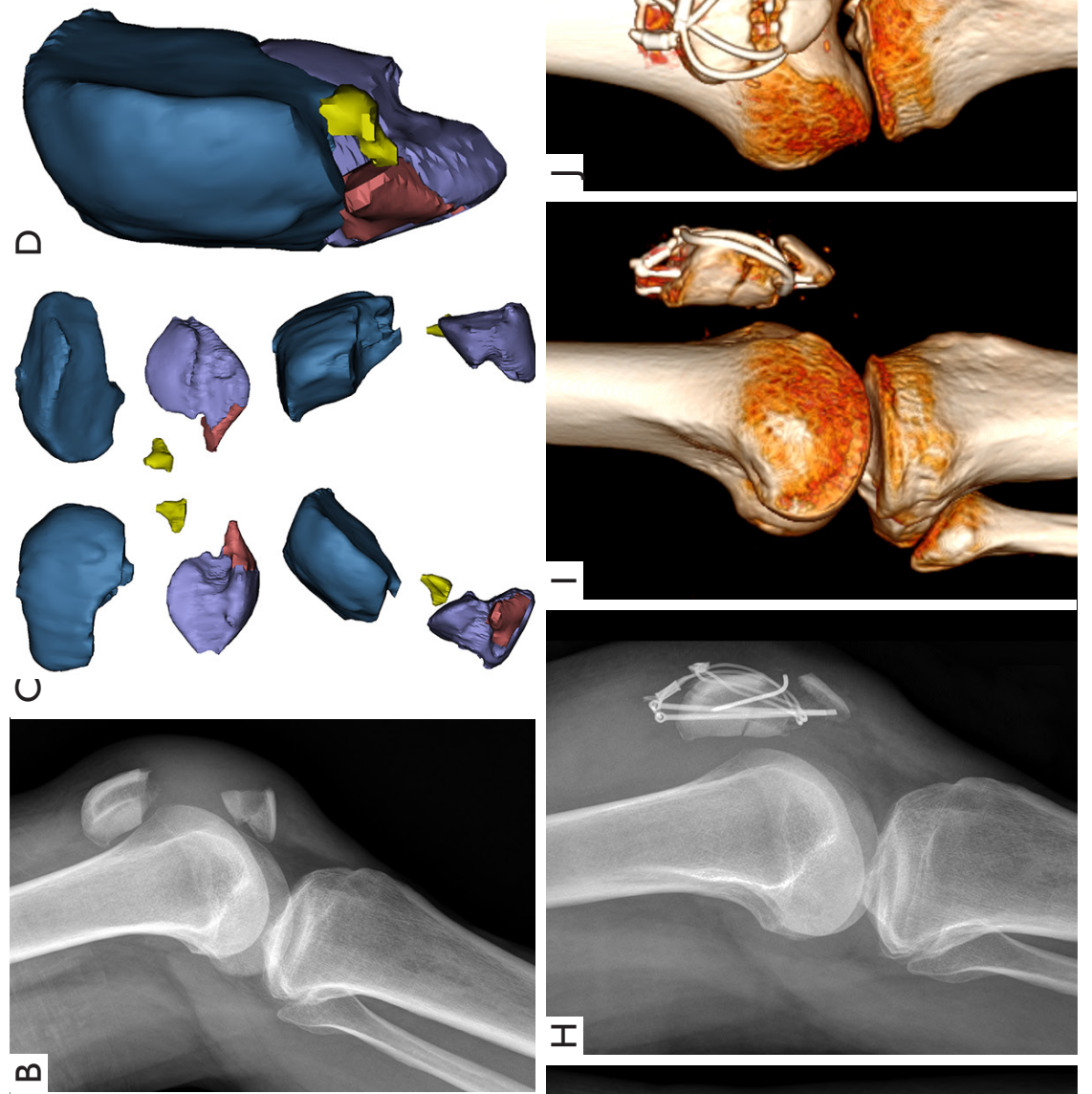

节节

ปี

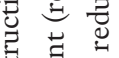

苟言

苍苛

क

w

芶

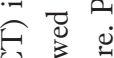

บ

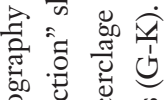

苛总

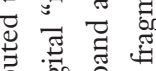
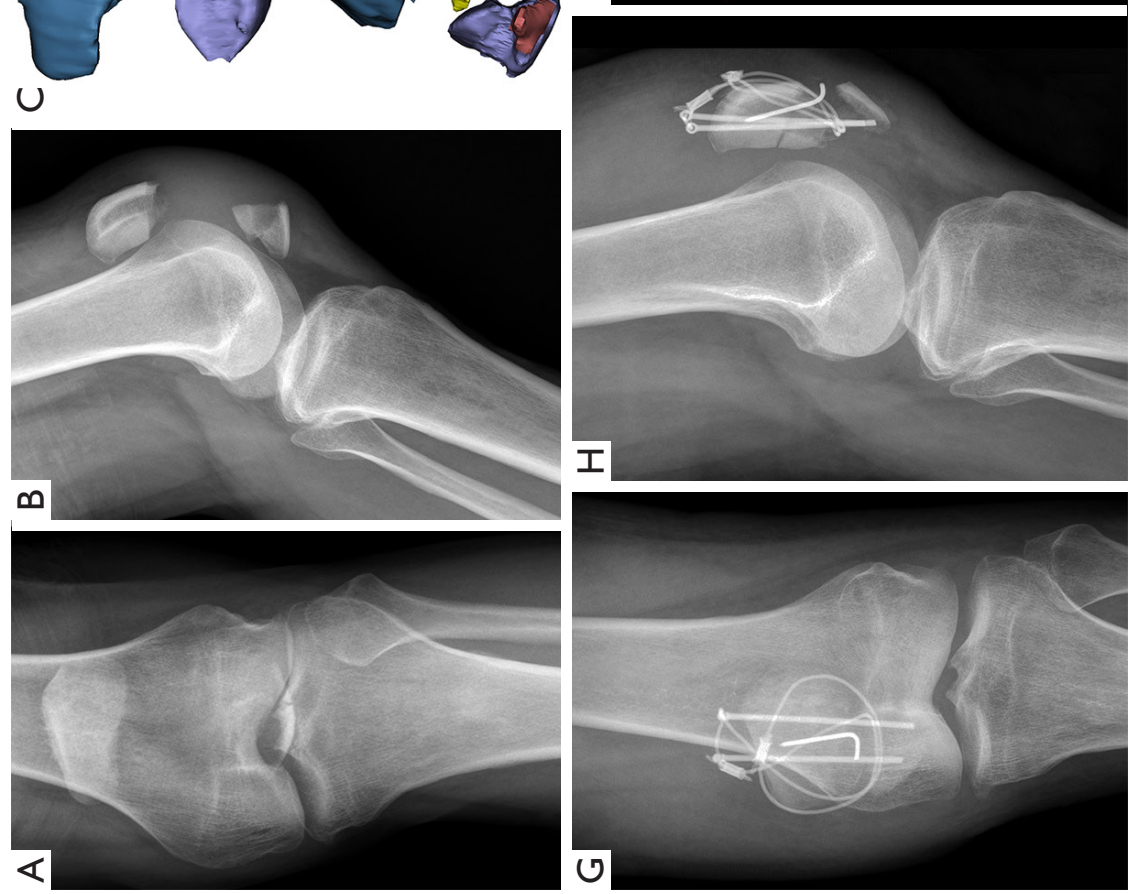

言

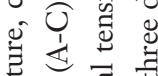

苞苛节

冚苞节

䒕 $\stackrel{0}{=}$

ㄹ. 융

预 壳

롱

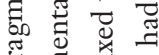

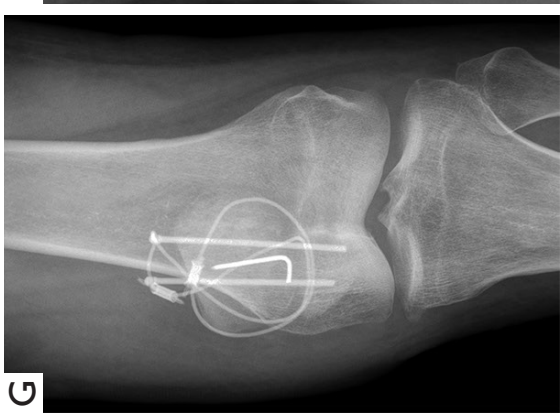

节造

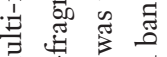

范壱当

( $)$ บ

言吾吉

牙

I 5 .

人

$\checkmark \stackrel{2}{*}$

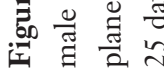


anterior plate osteosynthesis characterized by multiplanar fixation. These fracture maps could also be useful in biomechanical studies and development of new implants.

\section{Acknowledgments}

Funding: This work was supported by AOTrauma Asia Pacific of the AO Foundation [No. AOTAP20-22].

\section{Footnote}

Reporting Checklist: The authors have completed the STROBE reporting checklist. Available at https://dx.doi. org/10.21037/atm-21-1814

Data Sharing Statement: Available at https://dx.doi. org/10.21037/atm-21-1814

Peer Review File: Available at https://dx.doi.org/10.21037/ atm-21-1814

Conflicts of Interest: All authors have completed the ICMJE uniform disclosure form (available at https://dx.doi. org/10.21037/atm-21-1814). The authors report that this study was granted by AOTrauma Asia Pacific of the AO Foundation to reveal the morphologic details of the multifragmentary patella fracture (AO/OTA-34C3).

Ethical Statement: The authors are accountable for all aspects of the work in ensuring that questions related to the accuracy or integrity of any part of the work are appropriately investigated and resolved. The study was conducted in accordance with the Declaration of Helsinki (as revised in 2013). The study was approved by Institutional Ethics Committee of Shanghai Sixth People's Hospital (No.: 2016-89-1) and individual consent for this retrospective analysis was waived.

Open Access Statement: This is an Open Access article distributed in accordance with the Creative Commons Attribution-NonCommercial-NoDerivs 4.0 International License (CC BY-NC-ND 4.0), which permits the noncommercial replication and distribution of the article with the strict proviso that no changes or edits are made and the original work is properly cited (including links to both the formal publication through the relevant DOI and the license). See: https://creativecommons.org/licenses/by-nc-nd/4.0/.

\section{References}

1. Boström A. Fracture of the patella. A study of 422 patellar fractures. Acta Orthop Scand Suppl 1972;143:1-80.

2. Mahmoud MO. Patella. In: Buckley RE, Moran CG, Apivatthakakul T, editors. AO Principles of Fracture Management: Vol. 1: Principles, Vol. 2: Specific fractures. Stuttgart: Georg Thieme Verlag, 2018:853-64.

3. Matthews B, Hazratwala K, Barroso-Rosa S. Comminuted Patella Fracture in Elderly Patients: A Systematic Review and Case Report. Geriatr Orthop Surg Rehabil 2017;8:135-44.

4. Sim JA, Joo YB, Choi W, et al. Patellar fractures in elderly patients: a multicenter computed tomography-based analysis. Arch Orthop Trauma Surg 2020. [Epub ahead of print]. doi: 10.1007/s00402-020-03526-z.

5. Miller MA, Liu W, Zurakowski D, et al. Factors predicting failure of patella fixation. J Trauma Acute Care Surg 2012;72:1051-5.

6. Siljander M, Koueiter DM, Gandhi S, et al. Outcomes Following Low-Profile Mesh Plate Osteosynthesis of Patella Fractures. J Knee Surg 2018;31:919-26.

7. Moore TB, Sampathi BR, Zamorano DP, et al. Fixed angle plate fixation of comminuted patellar fractures. Injury 2018;49:1203-7.

8. Singer MS, Halawa AM, Adawy A. Outcome of low profile mesh plate in management of comminuted displaced fracture patella. Injury 2017;48:1229-35.

9. Xie X, Zhan Y, Dong M, et al. Two and ThreeDimensional CT Mapping of Hoffa Fractures. J Bone Joint Surg Am 2017;99:1866-74.

10. Xie X, Zhan Y, Wang Y, et al. Comparative Analysis of Mechanism-Associated 3-Dimensional Tibial Plateau Fracture Patterns. J Bone Joint Surg Am 2020;102:410-8.

11. Meinberg EG, Agel J, Roberts CS, Karam MD, Kellam JF. Fracture and Dislocation Classification Compendium-2018. J Orthop Trauma 2018;32 Suppl 1:S1-S170.

12. Zhang Y, Sun Y, Liao S, et al. Three-Dimensional Mapping of Medial Wall in Unstable Pertrochanteric Fractures. Biomed Res Int 2020;2020:8428407.

13. Rockwood and Green's Fractures in Adults. Available online: https://www.cengage.com/search/ productOverview.do;jsessionid=022F0D631CE43D829B4 B321C7CD09FA3?N=197+4294888968\&Ntk=P_EPI\&N $\mathrm{tt}=1299170681324635364966125708168529698 \& \mathrm{Ntx}=\mathrm{mo}$ de\%2Bmatchallpartial\&showMoreIds $=6$

14. Jarraya M, Diaz LE, Arndt WF, et al. Imaging of patellar 
fractures. Insights Imaging 2017;8:49-57.

15. Lazaro LE, Wellman DS, Pardee NC, et al. Effect of computerized tomography on classification and treatment plan for patellar fractures. J Orthop Trauma 2013;27:336-44.

16. Gwinner C, Märdian S, Schwabe P, et al. Current concepts review: Fractures of the patella. GMS Interdiscip Plast Reconstr Surg DGPW 2016;5:Doc01.

17. Misir A, Kizkapan TB, Uzun E, et al. Fracture Patterns and Comminution Zones in OTA/AO 34C Type Patellar Fractures. J Orthop Trauma 2020;34:e159-64.

18. Kfuri M, Escalante I, Schopper C, et al. Comminuted patellar fractures: The role of biplanar fixed angle plate constructs. J Orthop Translat 2021;27:17-24.

19. Matejčić A, Ivica M, Jurišić D, et al. Internal fixation of patellar apex fractures with the basket plate: 25 years of experience. Injury 2015;46 Suppl 6:S87-90.

Cite this article as: Zhan Y, Zhang Y, Xie X, Luo C. Threedimensional fracture mapping of multi-fragmentary patella fractures (AO/OTA 34C3). Ann Transl Med 2021;9(17):1364. doi: $10.21037 / \mathrm{atm}-21-1814$
20. Cho JW, Kim J, Cho W'T, et al. Comminuted inferior pole fracture of patella can be successfully treated with rimplate-augmented separate vertical wiring. Arch Orthop Trauma Surg 2018;138:195-202.

21. Zderic I, Stoffel K, Sommer C, et al. Biomechanical evaluation of the tension band wiring principle. A comparison between two different techniques for transverse patella fracture fixation. Injury 2017;48:1749-57.

22. Thelen S, Schneppendahl J, Jopen E, et al. Biomechanical cadaver testing of a fixed-angle plate in comparison to tension wiring and screw fixation in transverse patella fractures. Injury 2012;43:1290-5.

23. Wagner FC, Neumann MV, Wolf S, et al. Biomechanical comparison of a $3.5 \mathrm{~mm}$ anterior locking plate to cannulated screws with anterior tension band wiring in comminuted patellar fractures. Injury 2020;51:1281-7. 